\title{
Capacity of endometrial thickness measurement to diagnose endometrial carcinoma in asymptomatic postmenopausal women: a systematic review and meta-analysis
}

\author{
Dan $\mathrm{Su}^{1,2 \#}$, Ling $\mathrm{Li}^{2,3 \#}$, Min Zhong ${ }^{2,4}$, Yidan $\mathrm{Xia}^{1,2}$ \\ ${ }^{1}$ Department of Gynecology and Obstetrics, Sichuan Provincial People's Hospital, University of Electronic Science and Technology of China, \\ Chengdu, China; ${ }^{2}$ Chinese Academy of Sciences Sichuan Translational Medicine Research Hospital, Chengdu, China; ${ }^{3}$ Department of Ultrasound, \\ Sichuan Provincial People's Hospital, University of Electronic Science and Technology of China, Chengdu, China; ${ }^{4}$ Department of Operating Room, \\ Sichuan Provincial People's Hospital, University of Electronic Science and Technology of China, Chengdu, China \\ Contributions: (I) Conception and design: M Zhong, Y Xia; (II) Administrative support: Y Xia; (III) Provision of study materials or patients: D Su, L \\ Li; (IV) Collection and assembly of data: D Su, L Li; (V) Data analysis and interpretation: D Su, Y Xia; (VI) Manuscript writing: All authors; (VII) \\ Final approval of manuscript: All authors. \\ "These authors contributed equally to this work. \\ Correspondence to: Min Zhong; Yidan Xia. Sichuan Provincial People's Hospital, University of Electronic Science and Technology of China, Chengdu \\ 610072, China; Chinese Academy of Sciences Sichuan Translational Medicine Research Hospital, 32 West Second Section, First Ring Road, \\ Chengdu 610072, China. Email: 1468629056@qq.com; xiayd2002@163.com.
}

Background: Endometrial thickness (ET) is closely related to many gynecological symptoms. The measurement of ET is also an important tool for evaluating adverse symptoms such as bleeding in postmenopausal women. However, the significance of ET in asymptomatic women is still unclear. The purpose of this study was to determine the correlation between ET and the incidence of endometrial lesions in asymptomatic women after menopause, and to statistically analyze the correlation with a $5 \mathrm{~mm}$ cutoff value.

Methods: A systematic literature search was conducted in May 2021 to screen out articles that reported that ET measurement was used to diagnose endometrial carcinoma (EC), endometrial hyperplasia (EH), and endometrial polyps (EP) in asymptomatic postmenopausal women who did not use hormone replacement therapy (HRT). The endometrial membrane was set at $5 \mathrm{~mm}$ as the cut-off, and using $5 \mathrm{~mm}$ as the cut-off of the ET, the relationship between the thickness of the endometrium and the prevalence of EC, EH, and EP was evaluated. Relative risk (RR) and standardized mean difference (SMD) were extrapolated with $95 \%$ confidence interval (CI).

Results: After screening, 9 studies reported a total of 3,620 cases of asymptomatic postmenopausal women whose ET was measured. Among them, there were 1,758 cases of ET $<5 \mathrm{~mm}$, the probability of EC, EH, and EP were $0.284 \%(5 / 1,758), 0.398 \%$ (7/1,758), and 0.626\% (11/1,758), respectively. In another 1,862 cases with ET $\geq 5 \mathrm{~mm}$, the probabilities of EC, EH, and EP were 1.128\% (21/1,862), 1.128\% (21/1,862), and $1.557 \%$ (29/1,862), respectively. The results showed that ET can be used as a risk factor for predicting EC and other pathological changes.

Discussion: The results of this meta-analysis show that when the ET is greater than $5 \mathrm{~mm}$, the incidence of EC, EH, and EP increases significantly. It is reasonable to use ET as a screening test for EC and EH in asymptomatic postmenopausal women.

Keywords: Endometrial carcinoma (EC); endometrial thickness (ET); endometrial hyperplasia (EH); asymptomatic postmenopausal women; meta-analysis

Submitted Aug 16, 2021. Accepted for publication Oct 02, 2021.

doi: 10.21037/apm-21-2516

View this article at: https://dx.doi.org/10.21037/apm-21-2516 


\section{Introduction}

Endometrial carcinoma (EC) is the most common gynecological malignant tumor in developed countries such as Europe and the United States. It is the second most common malignant tumor of the female reproductive system in China, surpassed only by cervical cancer. The incidence rate in some developed cities such as Shanghai has gradually taken first place in gynecological malignancies (1). The main cause of death of endometrial cancer is failure to diagnose early, late stage anemia, weight loss, cachexia and metastasis, as well as clear cell carcinoma, serous carcinoma and other malignant pathological types. In recent years, thanks to the popularity of cervical cancer screening programs in the general population, the incidence of cervical cancer has declined. However, with the increase in life expectancy and the improvement of living standards, the incidence of EC has continued to increase and is showing a trend of younger generation. Screening and early diagnosis of EC remain unresolved clinical and epidemiological problems.

The significance of asymptomatic endometrial thickening is still unclear. Due to the lack of diagnosis and treatment standards, there is still much controversy about how clinicians should manage it. In recent years, a large number of studies have confirmed that the endometrial thickness (ET) of patients with postmenopausal vaginal bleeding has a significant correlation with the risk of EC (2). For asymptomatic women after menopause, there is no clear research report on the correlation between ET and $\mathrm{EC}$ risk, and there is no reasonable threshold to predict the correlation between ET and EC risk. Therefore, it is of great significance to explore the correlation between the ET and endometrial malignant transformation of asymptomatic women after menopause, so as to solve the clinical question of what ET should constitute the threshold for requiring further examination.

Transvaginal ultrasonography (TVS) is a simple and noninvasive method to examine the endometrium $(3,4)$, which can measure the ET and has a high negative predictive value for endometrial malignancy. It has become the first choice for patients with postmenopausal bleeding (PMB). Most of the current guidelines recommend that the ET of women with $\mathrm{PMB}$ is 4 or $5 \mathrm{~mm}$. When the ET is lower than this threshold, the risk of $\mathrm{EC}$ is less than $1 \%(5,6)$. There are many opinions on the ET related to EC, such as 5, 6, 8, 10, and $11 \mathrm{~mm}$ (7). The American College of Obstetricians and Gynecologists (ACOG) does not recommend routine endometrial biopsy after menopause with asymptomatic ET >4 mm (8). The Society of Obstetrics and Gynecology of Canada (SOGC) defines asymptomatic endometrial thickening as postmenopausal women without vaginal bleeding, ultrasound examination revealing ET of $>5 \mathrm{~mm}$, and pointed out that women with thickened endometrium should have other positive results on ultrasound. Findings such as increased blood vessel formation, uneven endometrium, granular fluid, and ET of more than $11 \mathrm{~mm}$ should be referred to a gynecologist for further examination (9). Some researchers believe that the prevalence of EC in asymptomatic postmenopausal women is lower than that of women with PMB, and the threshold of ET should be appropriately increased $(10,11)$. Therefore, for women with asymptomatic endometrial thickening after menopause, there is currently no clear ET threshold that can predict the risk of malignant transformation.

In order to study the correlation between the ET of asymptomatic women after menopause and the degree of endometrial malignancy, and to determine the critical value of endometrial thickening that requires further examination, we collected and recorded the ET of asymptomatic women after menopause. We performed meta-analysis the articles that diagnosed EC, endometrial hyperplasia $(\mathrm{EH})$, and endometrial polyps (EP). We present the following article in accordance with the Preferred Reporting Items for Systematic Reviews and Meta-Analyses (PRISMA) reporting checklist (available at https://dx.doi.org/10.21037/apm-21-2516).

\section{Methods}

\section{Search strategy}

In May 2021, we conducted an electronic search in the databases of Embase, PubMed, Web of Science, Cochrane Library, Wanfang, and China National Knowledge Infrastructure (CNKI). We searched for all published reports on the ET of asymptomatic postmenopausal women, or articles on EC, hyperplasia, and polyps. The following keywords were used: postmenopausal, asymptomatic, endometrial, thickness, hyperplasia, EC, polyps, ultrasound, and screening. The search was conducted without language restrictions, and abstracts or articles written in languages other than English and Chinese were translated by professional native language speakers. The references of the selected studies were searched for articles that were not identified by the electronic search, and the process was 
repeated for any further relevant research which was found. There was no registration review agreement.

\section{Inclusion criteria}

The inclusion criteria were as follows: research on the relationship between ET and EC in asymptomatic postmenopausal women published in the above databases from database inception to May 2021. Prospective or retrospective study, participants were postmenopausal women (menstruation stopped completely for at least 12 months) who were not using hormone replacement therapy (HRT), had no vaginal bleeding, discharge, increased vaginal discharge, and other symptoms. The measurement of ET was that of the double-layer ET at the thickest part of the sagittal section of the midline of the uterus. If the study recorded the value of the single-layer ET, the measurement was doubled and included. Availability of histological or cytological endometrial examination results, and the endometrial acquisition methods were not limited, including curettage, endometrial biopsy after hysterectomy, and uterine cavity endometrial biopsy under the microscope as acceptable. The data of the study were complete, and the average ET and standard deviation (SD) or standard error (SE) of asymptomatic postmenopausal women could be extracted.

\section{Exclusion criteria}

The exclusion criteria were as follows: case reports, abstracts, conferences, or reviews. The participants were PMB patients or women who were using HRT or other hormone analogs such as tamoxifen. The data provided in the article was incomplete, and only some participants had undergone histological verification studies. Vaginal ultrasound not only studied the diagnostic value of ET, but also excluded those involving uterine effusion, uneven endometrial echo, and so on. For repeated use of literature published by the same population, the largest number of samples were selected for inclusion in the study, and the rest were excluded.

\section{Paper screening and quality assessment}

Article selection was based on the PRISMA standard. After using EndNote X9 software to find duplicates in the literature, two people read the titles and/or abstracts to screen the studies that met the inclusion and exclusion criteria. Subsequently, full-text reading assessment was performed to determine final inclusion in the analysis.
Any dispute in the screening process was resolved through negotiation, or arbitration by a third party.

\section{Data extraction}

Data extraction for included studies, including author, publication time, country of origin, total sample size, method of obtaining uterine lining, cut-off value of ET, and number of participants with EC, EH, and EP. In order to estimate the range of normal ET in asymptomatic postmenopausal women, the average ET and SD or SE of the included study were extracted. In order to study the predictive accuracy of ET in the diagnosis of endometrial malignant tumors, it was necessary to extract the data required for corresponding sensitivity, specificity, negative predictive value, and positive predictive value. There have been studies that $5 \mathrm{~mm}$ can be used as the cutoff value of endometrial lesions (8), so this article will use $5 \mathrm{~mm}$ as the cutoff for research and analysis.

\section{Statistical analysis}

The general inverse variance method in RevMan 5.3 software (The Cochrane Collaboration, 2014) was used to calculate the combined average ET and $95 \%$ confidence interval (CI). The software Meta-DiSc 1.4 (Zamora et al., 2006) was used to calculate the effect indicators required for this meta-analysis, such as combined sensitivity, specificity, negative likelihood ratio, positive likelihood ratio, diagnostic odds ratio (OR), and so on, to draw the summary receiver operating characteristic curve (SROC) and calculate the area under the curve (AUC), and the results are presented in a forest map. If $\mathrm{P}>0.05, \mathrm{I}^{2}<50 \%$, it indicated that the heterogeneity of the included studies was low, and the fixed effects model was used for analysis; if $\mathrm{P}<0.05, \mathrm{I}^{2}>50 \%$, it indicated that there was heterogeneity that could not be ignored, and the random effects model analysis was used. By eliminated individual studies one by one, observed whether the results of the meta-analysis have essential changes. If there is no essential change, the results was highly reliable, and sensitivity analysis is carried out on this basis.

\section{Results}

\section{Search results and study characteristics}

According to the established search strategy, a total of 391 articles were retrieved, including 163 English language 


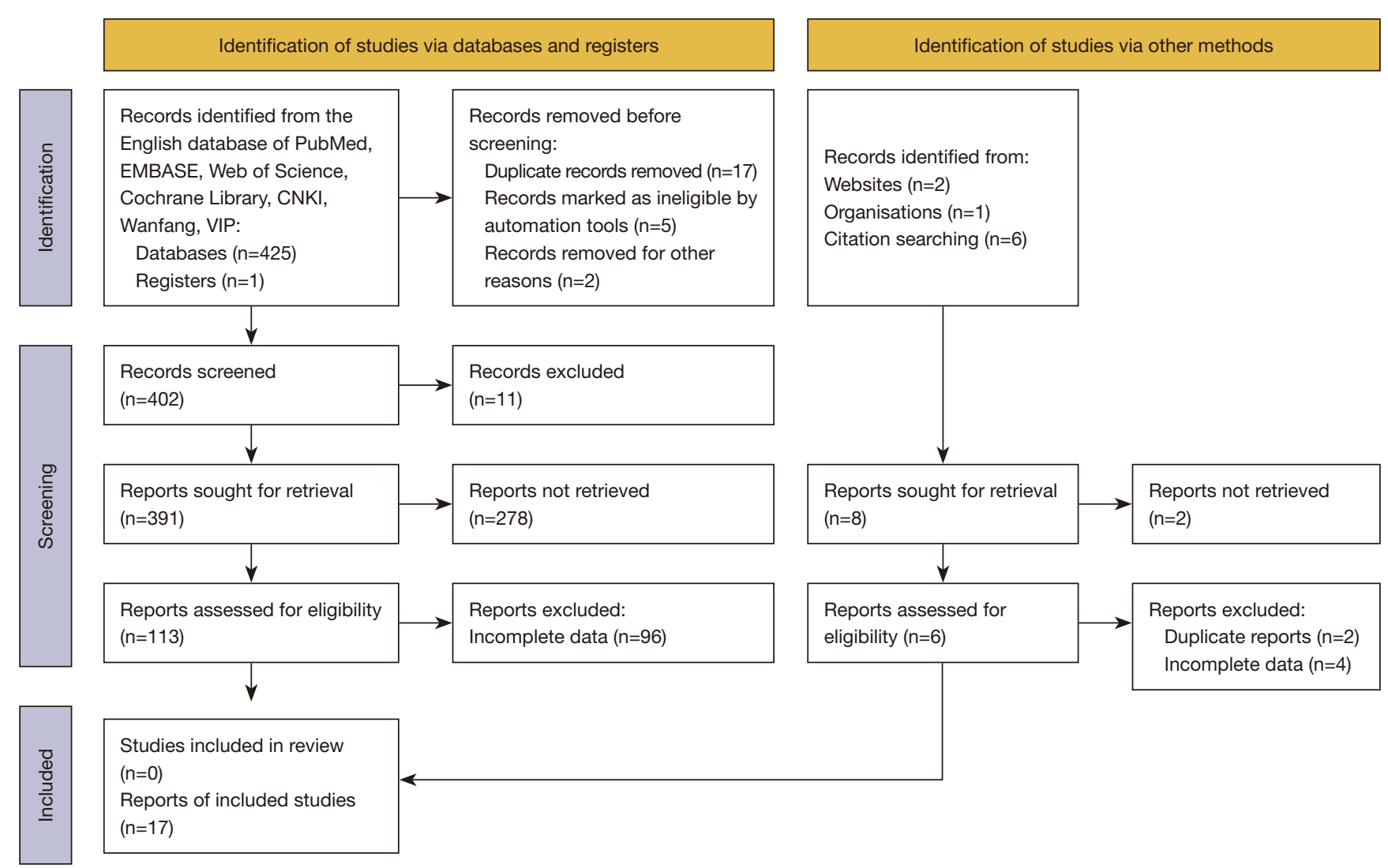

Figure 1 Flow chart of research included in the meta-analysis. CNKI, China National Knowledge Infrastructure.

articles and 228 Chinese language articles. Obviously irrelevant articles, duplicate articles, reviews, and so on were removed according to the inclusion and exclusion criteria, and 113 articles were initially screened. After reading the full text, we eliminated 96 articles from which complete data could not be extracted, and finally selected 17 articles to be included in this study. The specific search and screening process is shown in Figure 1. Among them, 8 articles could be used to estimate the thickness of the endometrium, a total of 4,175 patients were included in these studies, and the names of authors and publication dates of all studies are summarized in Table 1. A total of 9 articles could be used to analyze the accuracy of the diagnosis of EC, EH, and EP by the thickness of the endometrium, 3,620 patients were included in these studies, and the names of authors and publication dates of all studies are summarized in Table 2 .

\section{Meta-analysis results}

\section{ET in asymptomatic women after menopause}

The pooled estimates of the average ET of 4,175 women in the remaining 8 studies were $2.98 \mathrm{~mm}$ (95\% CI: 2.56 to $3.41 \mathrm{~mm})$. As $\mathrm{P}<0.05, \mathrm{I}^{2}>50 \%$, heterogeneity was indicated between the studies, thus the random effects model was adopted.

\section{EC}

These 9 studies included 3,620 postmenopausal women, of which 5 out of 1,758 cases with ET $<5 \mathrm{~mm}$ had EC, and 21 out of 1,862 cases with ET $\geq 5 \mathrm{~mm}$ had EC. Using a fixed-effects model, the pooled analysis showed that the difference in the incidence of EC between the two groups was statistically significant. The incidence of EC in ET $<5 \mathrm{~mm}$ was $0.284 \%(5 / 1,758)$, and the incidence of ET $\geq 5 \mathrm{~mm}$ was $1.128 \%(21 / 1,862)$ (OR $=0.35,95 \%$ CI: 0.16 to $\left.0.78, \mathrm{P}=0.01, \mathrm{I}^{2}=0 \%\right)$, as shown in Figure 2.

\section{EH}

A total of 7 out of 1,758 postmenopausal women with ET $<5 \mathrm{~mm}$ had EH. However, among 1,862 postmenopausal women with ET $\geq 5 \mathrm{~mm}, 21$ had EH. Through heterogeneity analysis, $\mathrm{I}^{2}=0 \%, \mathrm{P}=0.99$, so a fixed-effects 
Table 1 Characteristics of studies on ET

\begin{tabular}{|c|c|c|c|c|c|c|c|}
\hline Author & Country & Year & Journal & $\mathrm{N}$ & Average value & SD & Range \\
\hline Malinova et al. (12) & Bulgaria & 1996 & Int J Gynaecol Obstet & 130 & 3.86 & 2.35 & $1-28$ \\
\hline Gull et al. (13) & Sweden & 1996 & Ultrasound Obstet Gynecol & 361 & 3.00 & 1.9 & $1-17$ \\
\hline Warming et al. (14) & Denmark & 2002 & Ultrasound Obstet Gynecol & 1,182 & 2.10 & 1.4 & $1-18$ \\
\hline Kasraeian et al. (15) & Iran & 2011 & Climacteric & 259 & 3.83 & 2.95 & $1-15$ \\
\hline Nakamura et al. (16) & Japan & 2014 & SA J Radiol & 297 & 2.4 & 0.1 & $0.2-25$ \\
\hline Hartman et al. (2) & Canada & 2013 & Gynecol Obstet Invest & 1,500 & 3.71 & 1.9 & $0.2-13$ \\
\hline
\end{tabular}

ET, endometrial thickness; SD, standard deviation.

Table 2 Studies characteristics of the relationship between ET and EC

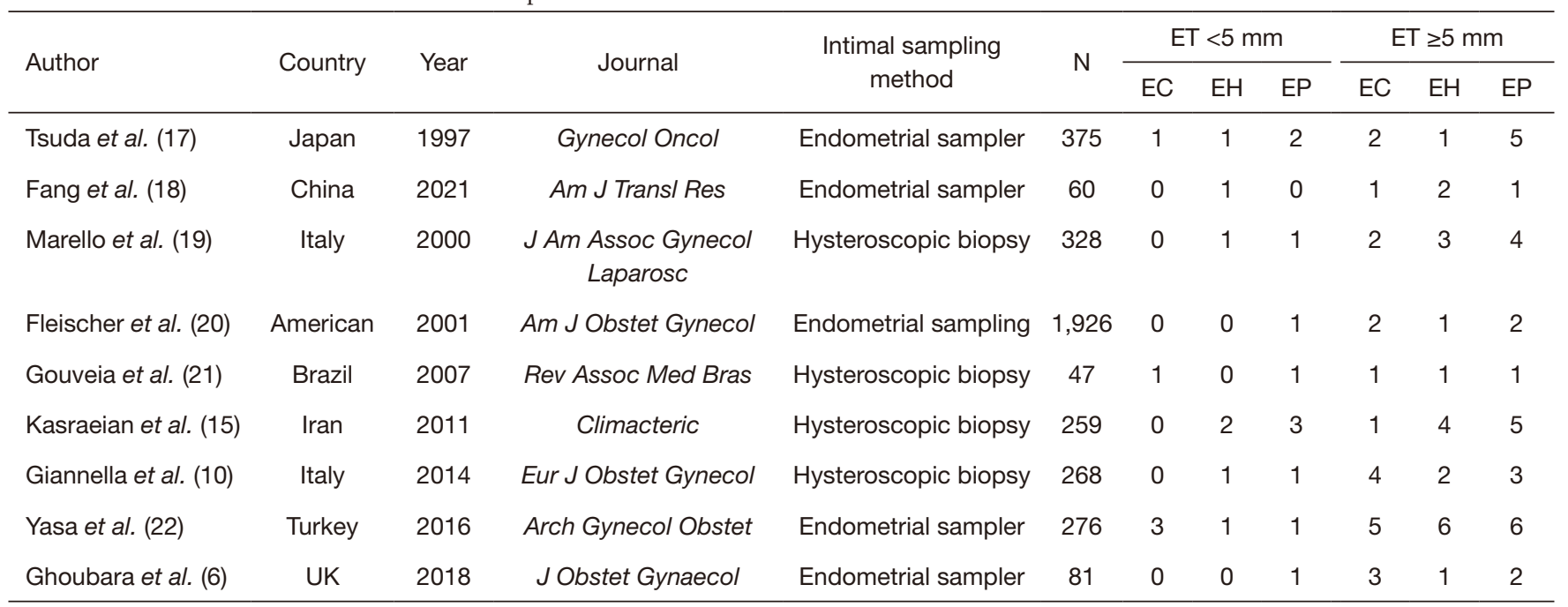

$\mathrm{ET}$, endometrial thickness; EC, endometrial carcinoma; EH, endometrial hyperplasia; EP, endometrial polyps.

model was used. Meta-analysis results showed that there was an association between ET $\geq 5 \mathrm{~mm}$ and $\mathrm{EH}$. The incidence of $\mathrm{EH}$ in $\mathrm{ET}<5 \mathrm{~mm}$ was $0.398 \%(7 / 1,758)$, and the incidence of $\mathrm{ET} \geq 5 \mathrm{~mm}$ was $1.128 \%(21 / 1,862)$ (OR $=0.39,95 \%$ CI: 0.18 to $0.87, \mathrm{P}=0.02, \mathrm{I}^{2}=0 \%$ ), as shown in Figure 3.

\section{EP}

Among the 1,758 postmenopausal women whose ET was $<5 \mathrm{~mm}, 11 \mathrm{had}$ EP. Among the other 1,862 postmenopausal women with ET $\geq 5 \mathrm{~mm}, 29$ had EP. The heterogeneity analysis showed that $\mathrm{I}^{2}=0 \%, \mathrm{P}=0.98$, so a fixed-effects model was used, and the results of metaanalysis showed that $\mathrm{ET} \geq 5 \mathrm{~mm}$ was a risk factor for EP.
The incidence of EP in ET $<5 \mathrm{~mm}$ was $0.626 \%(11 / 1,758)$, and the incidence of ET $\geq 5 \mathrm{~mm}$ was $1.557 \%(29 / 1,862)$ $\left(\mathrm{OR}=0.41,95 \% \mathrm{CI}: 0.21\right.$ to $\left.0.81, \mathrm{P}=0.01, \mathrm{I}^{2}=0 \%\right)$, as shown in Figure 4.

\section{Publication bias}

The funnel chart was used to examine the publication bias of the relationship between ET and EC, EH, and EP. According to the visual inspection of the funnel chart, the three groups of funnel charts were almost symmetrical, and there was less publication bias than if the three funnel charts all showed asymmetry, indicating that there may be less publication bias when including adverse clinical endpoint events, as shown in Figure 5. 


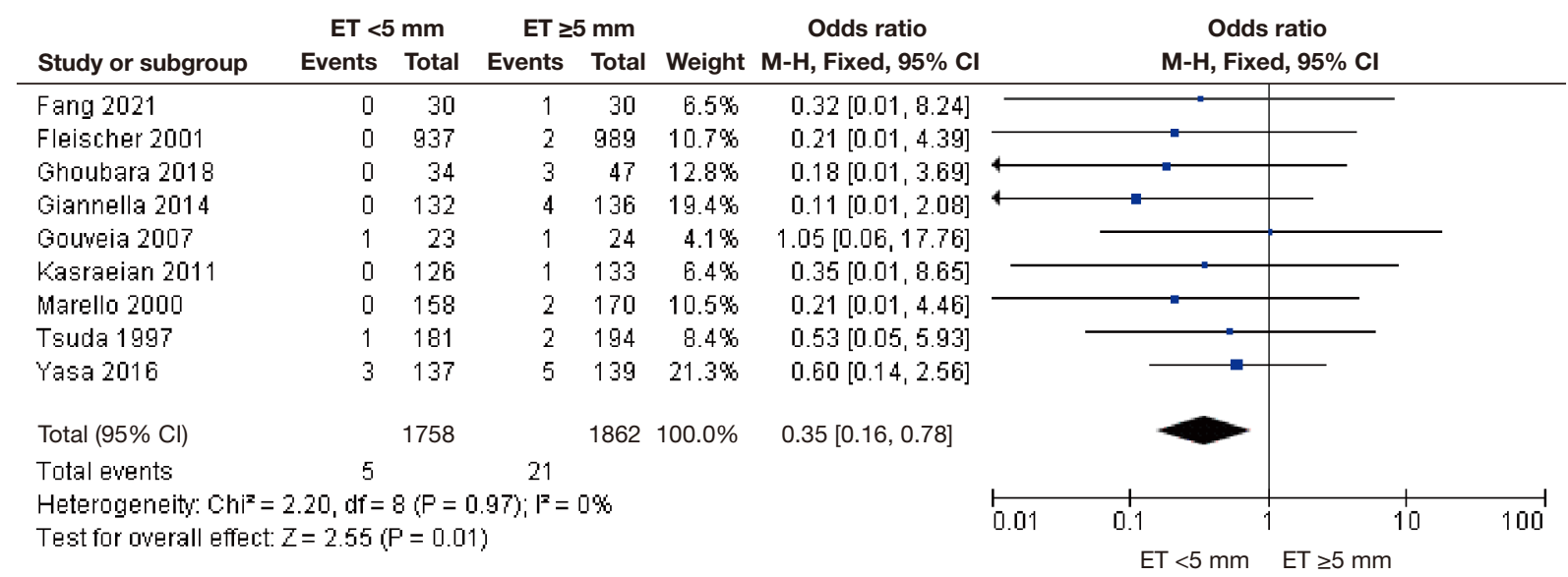

Figure 2 Meta-analysis forest plot of the relationship between ET and EC using a fixed-effect model. Comparison: the relationship between the ET and the incidence of EC. Statistical method: inverse variance of the fixed effects model (OR and 95\% CI). ET, endometrial thickness; EC, endometrial carcinoma; OR, odds ratio; CI, confidence interval.

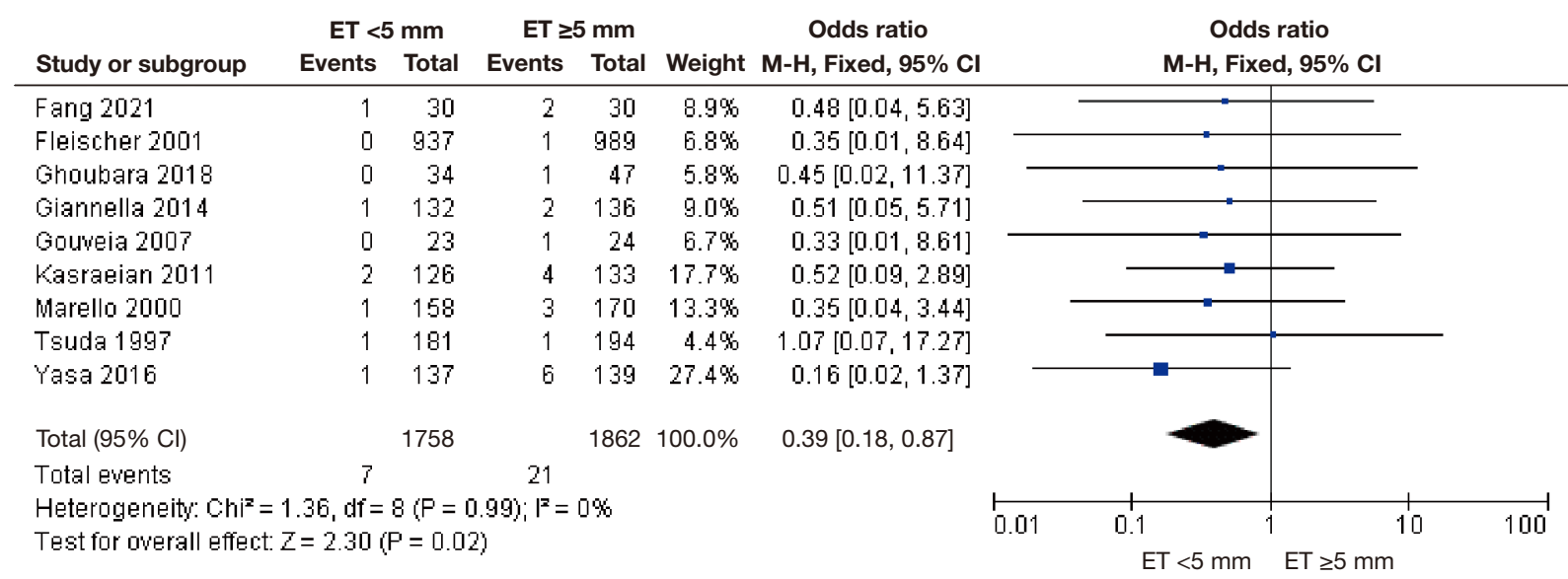

Figure 3 Meta-analysis forest plot of the relationship between ET and EH using a fixed-effect model. Comparison: the relationship between ET and the incidence of EH. Statistical method: inverse variance of the fixed effects model (OR and 95\% CI). ET, endometrial thickness; $\mathrm{EH}$, endometrial hyperplasia; OR, odds ratio; CI, confidence interval.

\section{Discussion}

The term EC is used to describe a group of epithelial malignant tumors that occur in the endometrium, and is 1 of the 3 major malignant tumors of the female reproductive tract. In the United States, the incidence of uterine body tumors is $7 \%$; after breast cancer, lung cancer, and colorectal cancer, it ranks 4th among female malignant tumors, and its mortality ranks 6th (23). In China, EC is not the most common malignant tumor of the female genital tract, and its incidence is lower than that of cervical cancer $(1,11)$. However, with the rapid development of modern society and economy, the accelerated process of population aging, and changes in lifestyles, the high-risk factors of EC such as obesity, overweight, and diabetes have increased annually, in tandem with the incidence rate of EC itself. While the incidence of EC has increased, it has also become younger, which seriously threatens women's lives and health (24). Therefore, the study of asymptomatic endometrial thickening after menopause is of great significance, and it may be particularly instructive for the screening and early diagnosis of EC.

For women with vaginal $\mathrm{PMB}$, a large number of studies 


\begin{tabular}{|c|c|c|c|c|c|c|c|c|c|c|}
\hline \multirow[b]{2}{*}{ Study or subgroup } & \multicolumn{2}{|c|}{$\mathrm{ET}<5 \mathrm{~mm}$} & \multicolumn{2}{|c|}{$\mathrm{ET} \geq 5 \mathrm{~mm}$} & \multicolumn{2}{|r|}{ Odds ratio } & \multirow{2}{*}{\multicolumn{4}{|c|}{$\begin{array}{l}\text { Odds ratio } \\
\text { M-H, Fixed, } 95 \% \mathrm{Cl}\end{array}$}} \\
\hline & Events & Total & Events & Total & Weight & M-H, Fixed, $95 \% \mathrm{Cl}$ & & & & \\
\hline Fang 2021 & 0 & 30 & 1 & 30 & $5.2 \%$ & $0.32[0.01,8.24]$ & & & & \\
\hline Fleischer 2001 & 1 & 937 & 2 & 989 & $6.9 \%$ & $0.53[0.05,5.82]$ & & & & \\
\hline Ghoubara 2018 & 1 & 34 & 2 & 47 & $5.8 \%$ & $0.68[0,06,7.84]$ & & & & \\
\hline Giannella 2014 & 1 & 132 & 3 & 136 & $10.4 \%$ & $0.34[0.03,3.30]$ & & & & \\
\hline Gouweia 2007 & 1 & 23 & 1 & 24 & $3.3 \%$ & $1.05[0.06,17.76]$ & & & & \\
\hline Kasraeian 2011 & 3 & 126 & 5 & 133 & $16.9 \%$ & $0.62[0.15,2.67]$ & & & & \\
\hline Marello 2000 & 1 & 158 & 4 & 170 & $13.6 \%$ & $0.26[0.03,2.39]$ & & & & \\
\hline Tsuda 1997 & 2 & 181 & 5 & 194 & $16.9 \%$ & $0.42[0.08,2.20]$ & & & & \\
\hline Yasa 2016 & 1 & 137 & 6 & 139 & $21.0 \%$ & $0.16[0,02,1.37]$ & & & + & \\
\hline Total (95\% Cl) & & 1758 & & 1862 & $100.0 \%$ & $0.41[0.21,0.81]$ & & & & \\
\hline Total events & 11 & & 29 & & & & & & & \\
\hline \multicolumn{7}{|c|}{$\begin{array}{l}\text { Heterogeneity: } \mathrm{Ch}^{2}=1.87, \mathrm{df}=8(\mathrm{P}=0.98) ; \mathrm{P}^{2}=0 \% \\
\text { Test for overall effect: } \mathrm{Z}=2.55(\mathrm{P}=0.01)\end{array}$} & 0.01 & 0.1 & 10 & 100 \\
\hline
\end{tabular}

Figure 4 Meta-analysis forest plot of the relationship between ET and EP using a fixed-effect model. Comparison: the relationship between ET and the incidence of EP. Statistical method: inverse variance of the fixed effects model (OR and 95\% CI). ET, endometrial thickness; EP, endometrial polyps; OR, odds ratio; CI, confidence interval.
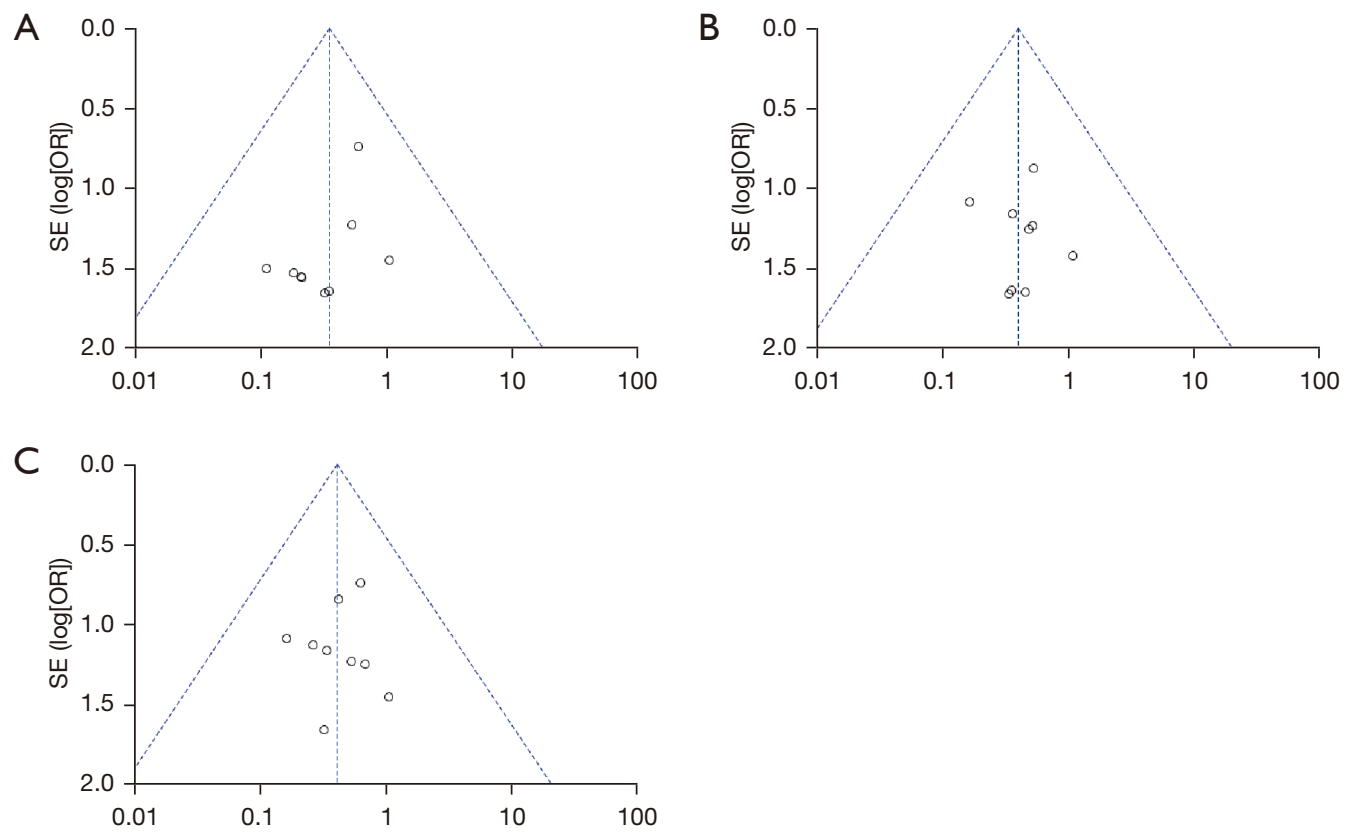

Figure 5 Publication bias is analyzed by funnel plot. Comparison of: EC (A), EH (B), and EP (C), respectively. EC, endometrial carcinoma; $\mathrm{EH}$, endometrial hyperplasia; EP, endometrial polyps; OR, odds ratio; SE, standard error.

have confirmed that the ET is significantly related to the risk of EC (25). Because TVS is noninvasive, simple, reproducible, and has high sensitivity and good negative predictive value, it has become the first choice for patients with PMB. An ET $\leq 4 \mathrm{~mm}$ is used as a reference and basis for the selection of diagnostic curettage biopsy for the diagnosis of EC. When the ET of postmenopausal women is less than or equal to $4 \mathrm{~mm}$, the accuracy of negative prediction can reach 99\%, and when the ET exceeds $5 \mathrm{~mm}$, the sensitivity is similar to that of endometrial biopsy (8). Therefore, in this study, $5 \mathrm{~mm}$ was chosen as the critical value of the study.

For asymptomatic women without vaginal bleeding, fluid flow, or leucorrhea after menopause, the relationship 
between $\mathrm{ET}$ and the risk of $\mathrm{EC}$ is not yet clear, and there is no reasonable ET threshold to predict EC risk. There are different opinions on the ET related to EC, such as 5, $6,8,10$, and $11 \mathrm{~mm}(7)$. The results of this study showed that the average ET of asymptomatic postmenopausal women was $2.98 \mathrm{~mm}$ (95\% CI: 2.56 to $3.41 \mathrm{~mm}$ ), which was consistent with the report by Breijer et al. (26). Among asymptomatic postmenopausal women who did not use hormone therapy, $77.1 \%$ had ET $<4 \mathrm{~mm}$, and only $8 \%$ had ET $>8 \mathrm{~mm}$ (2). Among the 3,620 postmenopausal women without vaginal bleeding, 1,758 had ET less than $5 \mathrm{~mm}$, there were 5 cases of EC, 7 cases of $\mathrm{EH}$, and 11 cases of EP. There were 1,862 cases of ET $\leq 5 \mathrm{~mm}, 21$ cases of EC and $\mathrm{EH}$, and 29 cases of EP. The significant difference indicated that the risk of malignant transformation still had a certain relationship with ET.

This study has some limitations, because the incidence of EC in asymptomatic people is low, and screening for intrauterine cancer is not universal, most of the included studies were retrospective studies or small sample studies, and were based on cut-off values. After grouping, the number of studies in each group was small, which reduces the credibility of the research results. To fully study the importance of endometrial thickening in postmenopausal women, it is necessary to conduct large-scale prospective trials on asymptomatic women, so that the results of metaanalysis can provide more reliable evidence-based medical evidence.

\section{Conclusions}

The results of this meta-analysis show that when the ET is greater than $5 \mathrm{~mm}$, the frequency of $\mathrm{EC}, \mathrm{EP}$, and $\mathrm{EH}$ is significantly increased. We therefore recommend using $5 \mathrm{~mm}$ as the cut-off value has high sensitivity for diagnosing all common types of endometrial lesions. Postmenopausal women with $\mathrm{ET}>5 \mathrm{~mm}$ have a significant prevalence of endometrial lesions such as EC and $\mathrm{EH}$.

\section{Acknowledgments}

Funding: None.

\section{Footnote}

Reporting Checklist: The authors have completed the PRISMA reporting checklist. Available at https://dx.doi. org/10.21037/apm-21-2516
Conflicts of Interest: All authors have completed the ICMJE uniform disclosure form (available at https://dx.doi. org/10.21037/apm-21-2516). The authors have no conflicts of interest to declare.

Ethical Statement: The authors are accountable for all aspects of the work in ensuring that questions related to the accuracy or integrity of any part of the work are appropriately investigated and resolved.

Open Access Statement: This is an Open Access article distributed in accordance with the Creative Commons Attribution-NonCommercial-NoDerivs 4.0 International License (CC BY-NC-ND 4.0), which permits the noncommercial replication and distribution of the article with the strict proviso that no changes or edits are made and the original work is properly cited (including links to both the formal publication through the relevant DOI and the license). See: https://creativecommons.org/licenses/by-nc-nd/4.0/.

\section{References}

1. Chen $W$, Zheng R, Baade PD, et al. Cancer statistics in China, 2015. CA Cancer J Clin 2016;66:115-32.

2. Hartman A, Wolfman W, Nayot D, et al. Endometrial thickness in 1,500 asymptomatic postmenopausal women not on hormone replacement therapy. Gynecol Obstet Invest 2013;75:191-5.

3. Minagawa $\mathrm{Y}$, Sato S, Ito M, et al. Transvaginal ultrasonography and endometrial cytology as a diagnostic schema for endometrial cancer. Gynecol Obstet Invest 2005;59:149-54.

4. Cong Q, Luo L, Fu Z, et al. Histopathology of women with non-uniform endometrial echogenicity and risk factors for atypical endometrial hyperplasia and carcinoma. Am J Transl Res 2021;13:4500-9.

5. Ozer A, Ozer S, Kanat-Pektas M. Correlation between transvaginal ultrasound measured endometrial thickness and histopathological findings in Turkish women with abnormal uterine bleeding. J Obstet Gynaecol Res 2016;42:573-8.

6. Ghoubara A, Emovon E, Sundar S, et al. Thickened endometrium in asymptomatic postmenopausal women - determining an optimum threshold for prediction of atypical hyperplasia and cancer. J Obstet Gynaecol 2018;38:1146-9.

7. Jurkovic D, Alfirevic Z. DISQ 2: Endometrial thickness in asymptomatic women. Ultrasound Obstet Gynecol 
2005;26:203.

8. ACOG Committee Opinion No. 734: The role of transvaginal ultrasonography in evaluating the endometrium of women with postmenopausal bleeding. Obstet Gynecol 2018;131:e124-9.

9. Wolfman W. No. 249-Asymptomatic Endometrial Thickening. J Obstet Gynaecol Can 2018;40:e367-77.

10. Giannella L, Mfuta K, Setti T, et al. Diagnostic accuracy of endometrial thickness for the detection of intra-uterine pathologies and appropriateness of performed hysteroscopies among asymptomatic postmenopausal women. Eur J Obstet Gynecol Reprod Biol 2014;177:29-33.

11. Fan Y, Li X, Wang J, et al. Analysis of pregnancyassociated factors after fertility-sparing therapy in young women with early stage endometrial cancer or atypical endometrial hyperplasia. Reprod Biol Endocrinol 2021;19:118.

12. Malinova M, Pehlivanov B. Transvaginal sonography and progesterone challenge for identifying endometrial pathology in postmenopausal women. Int J Gynaecol Obstet 1996;52:49-53.

13. Gull B, Karlsson B, Milsom I, et al. Transvaginal sonography of the endometrium in a representative sample of postmenopausal women. Ultrasound Obstet Gynecol 1996;7:322-7.

14. Warming L, Ravn P, Skouby S, et al. Measurement precision and normal range of endometrial thickness in a postmenopausal population by transvaginal ultrasound. Ultrasound Obstet Gynecol 2002;20:492-5.

15. Kasraeian M, Asadi N, Ghaffarpasand F, et al. Value of transvaginal ultrasonography in endometrial evaluation of non-bleeding postmenopausal women. Climacteric 2011;14:126-31.

16. Nakamura J, Yoshikawa T, Maeda E, et al. Evaluation of endometrial thickness in postmenopausal women by using 3.0-T MRI. SA J Radiol 2014;18:1-4.

17. Tsuda H, Kawabata M, Yamamoto K, et al. Prospective study to compare endometrial cytology and transvaginal ultrasonography for identification of endometrial

Cite this article as: Su D, Li L, Zhong M, Xia Y. Capacity of endometrial thickness measurement to diagnose endometrial carcinoma in asymptomatic postmenopausal women: a systematic review and meta-analysis. Ann Palliat Med 2021;10(10):10840-10848. doi: 10.21037/apm-21-2516 malignancies. Gynecol Oncol 1997;65:383-6.

18. Fang F, Xu H, Wu L, et al. LNG-IUS combined with progesterone ameliorates endometrial thickness and pregnancy outcomes of patients with early-stage endometrial cancer or atypical hyperplasia. Am J Transl Res 2021;13:5412-9.

19. Marello F, Bettocchi S, Greco P, et al. Hysteroscopic evaluation of menopausal patients with sonographically atrophic endometrium. J Am Assoc Gynecol Laparosc 2000;7:197-200.

20. Fleischer AC, Wheeler JE, Lindsay I, et al. An assessment of the value of ultrasonographic screening for endometrial disease in postmenopausal women without symptoms. Am J Obstet Gynecol 2001;184:70-5.

21. Gouveia DA, Bahamondes L, Aldrighi JM, et al. Prevalence of endometrial injury in asymptomatic obese women. Rev Assoc Med Bras (1992) 2007;53:344-8.

22. Yasa C, Dural O, Bastu E, et al. Evaluation of the diagnostic role of transvaginal ultrasound measurements of endometrial thickness to detect endometrial malignancy in asymptomatic postmenopausal women. Arch Gynecol Obstet 2016;294:311-6.

23. Siegel RL, Miller KD, Fuchs HE, et al. Cancer statistics, 2021. CA Cancer J Clin 2021;71:7-33.

24. Rebegea L, Ilie AM, Neagu A, et al. Cutaneous and inguinal lymph nodes metastases disseminated from an endometrial serous carcinoma - case report and literature review. Chirurgia (Bucur) 2021;116:109-16.

25. Vetter MH, Smith B, Benedict J, et al. Preoperative predictors of endometrial cancer at time of hysterectomy for endometrial intraepithelial neoplasia or complex atypical hyperplasia. Am J Obstet Gynecol 2020;222:60.e1-7.

26. Breijer MC, Peeters JA, Opmeer BC, et al. Capacity of endometrial thickness measurement to diagnose endometrial carcinoma in asymptomatic postmenopausal women: a systematic review and meta-analysis. Ultrasound Obstet Gynecol 2012;40:621-9.

(English Language Editor: J. Jones) 